\title{
Trigeminal sensory neuropathy and mixed connective tissue disease: A case report
}

\author{
Tadashi Nakamura ${ }^{1}$, Mitsuharu Ueda $^{2}$, Hirokazu Takaoka ${ }^{1}$ and Yukio Ando ${ }^{2}$ \\ ${ }^{1}$ Section of Clinical Rheumatology, Kumamoto Shinto General Hospital, Kumaoto, Japan \\ ${ }^{2}$ Department of Neurology, Graduate School of Medical Sciences, Kumamoto University, Kumamoto, Japan
}

\begin{abstract}
Mixed connective tissue disease (MCTD), a multisystem disorder, has features of systemic lupus erythematosus, scleroderma, and polymyositis/dermatomyositis but also a high titer of antibody to ribonucleoprotein. A 62-year-old Japanese woman with MCTD had an isolated trigeminal sensory neuropathy as an early manifestation of MCTD. Raynaud's phenomenon occurred almost simultaneously with the neuropathy onset, followed by hand swelling, myositis, and increased ribonucleoprotein antibody. We herein describe our patient with trigeminal neuropathy as an early MCTD manifestation.
\end{abstract}

\section{Introduction}

Mixed connective tissue disease (MCTD) is a systemic autoimmune disease typifying by overlapping features between two or more systemic autoimmune diseases such as systemic lupus erythematosus (SLE), systemic sclerosis (SSc), and polymyositis/dermatomyositis (PM/DM) [1]. High titers of circulating antoantibodies against nuclear ribonucleoprotein (RNP) antigen, which are now referred to as anti-U1 RNP antibodies, are characteristic and justify the diagnosis of MCTD as a distinct clinical entity [2]. The hallmarks of this condition are Raynaud's phenomenon, swelling of the hands, arthralgia, lymphadenopathy, myalgia, and fatigue, all of which may develop over months or years. Sclerodactyly, myositis, puffy fingers, and swallowing defects are common. The issue about whether MCTD should be regarded as an independent disease has been solved: because of changing concepts, MCTD is now regarded as a distinct disease entity [3]. Although MCTD is thought to be associated with a relatively low incidence of renal disease and with an overall favorable prognosis, recent studies revealed a general trend toward more sclerodermalike features, pulmonary hypertension, and renal involvement $[4,5]$. Neurological manifestations rarely occur in MCTD, and the extent and exact nature of these manifestations are not well defined, with very few reports of trigeminal neuropathy occurring in MCTD [6-8]. However, we here report a case in which an isolated trigeminal sensory neuropathy occurred almost simultaneously with the rest of the clinical syndrome. We also discuss the development of such a neurological symptom associated with MCTD.

\section{Case report}

A 62-year-old Japanese woman was transferred to our hospital for evaluation of general malaise and persistent low-grade fever. She had been experiencing Raynaud's phenomenon, which occurred almost simultaneously with the onset of trigeminal neuropathy, myalgia in the upper extremities, diffuse swelling of the hands, and left shoulder pain, with the last beginning during the previous 2 weeks. She also had increased anti-RNP antibody titers. She had a temperature of $37.8^{\circ} \mathrm{C}$, regular heart rate of 78 beats/min, and blood pressure of $124 / 82 \mathrm{mmHg}$, and she was able to communicate well. The physical examination revealed unremarkable findings, except for the presence of indurated edema of the hands with sclerodactyly. The neurological examination indicated right trigeminal neuropathy, lethargy, and numbness of the right side of the face. The trigeminal nerve, i.e., the fifth $(\mathrm{V})$ cranial nerve, is responsible for sensation in the face via three branches: V1, $\mathrm{V} 2$, and V3. V1 is the ophthalmic branch, V2 is the maxillary branch, and V3 is the mandibular branch. Symptoms suggested that the trigeminal neuropathy was unilateral on the right side and resulted from effects of V2. She developed sensations of numbness and tingling affecting the right cheek. The numbness extended to the side of the nose, and she complained that the sensation was similar to the feeling resulting from a dental anesthetic. An examination revealed reduced sensory perception in the area supplied by V2. The jaw jerk, which is the motor component of $\mathrm{V} 2$, and the remainder of the nervous system were normal. No other neurological abnormalities were found, nor there was clinical evidence of muscle weakness in her face. Axial gadolinium-enhanced T1-weighted magnetic resonance imaging of the head showed no contrast enhancement in the cisternal part of the right trigeminal nerve. An isolated trigeminal sensory neuropathy was recognized as the early or initial manifestation of MCTD at admission.

Laboratory tests showed complete blood cell counts within normal limits; an increased erythrocyte sedimentation rate of 46 $\mathrm{mm} / \mathrm{h}$ (normal: 3-15); hypoalbuminemia of $3.7 \mathrm{~g} / \mathrm{dl}$ (normal: 4.1-5.1); elevated liver enzyme levels [aspartate aminotransferase value of 63 IU/L (normal: 13-34) and alanine aminotransferase value of $48 \mathrm{IU} / \mathrm{L}$ (normal: 7-37)]; increased lactate dehydrogenase value of $368 \mathrm{IU} / \mathrm{L}$ (normal: 112-213); elevated creatinine phosphokinase (CPK) value of

Correspondence to: Tadashi Nakamura, MD, $\mathrm{PhD}$, Section of Clinical Rheumatology, Kumamoto Shinto General Hospital, 1-17-27 Shinyashiki Chuo, Kumamoto 862-8655, Japan, Tel: +81 96364 6000; Fax: +81 96362 5204; E-mail: nakamura@k-shinto.or.jp

Key words: Anti-RNP antibody, mixed connective tissue disease, trigeminal neuropathy

Received: June 13, 2015; Accepted: July 02, 2015; Published: July 04, 2015 
298 IU/L (normal: 45-176); normal C-reactive protein level of $0.06 \mathrm{mg} /$ $\mathrm{dl}$ (normal: $<0.3$ ); elevated serum amyloid A protein value of $10.3 \mu \mathrm{g} / \mathrm{ml}$ (normal: < 8.0); and increased immunoglobulin G value of $3367 \mathrm{mg} / \mathrm{dl}$ (normal: 870-1700). Test results were positive for current antinuclear antibody at > X1280 (normal: < X40), speckled; anti-Sm antibody at $20.8 \mathrm{U} / \mathrm{ml}$ (normal: $<10$ ), anti-RNP antibody at $>500.0 \mathrm{U} / \mathrm{ml}$ (normal: $<10$ ), whereas test results were all normal for rheumatoid factor, anticyclic citrullinated peptide antibody, anti-double-stranded DNA immunoglobulin $\mathrm{G}$ antibody, anti-Scl-70 antibody, anti-Ro/SS-A, antiLo/SS-B antibody, anti-140-kDa polypeptide (CADM-140) antibody, anti-centromere antibody, and enzyme-linked immunosorbent assay for antibodies to proteinase- 3 and myeloperoxidase. Values of thyroid function and complement levels were within normal limits. Results of serological tests and polymerase chain reaction analyses for hepatitis B, hepatitis C, cytomegalovirus, and Epstein-Barr virus were normal. Urinalysis indicated no abnormalities. The chest X-ray showed a very slight reticular shadow at peripheral areas in both lungs. Whole body computed tomography suggested no malignant lesions, and biopsy of forearm skin gave a result that was identical to that of SSc, with loose arrangements of collagenous fibers in the dermis and atrophic epidermal connective tissue. MCTD and concurrent trigeminal isolated sensory neuropathy associated with MCTD were therefore diagnosed according to current diagnostic criteria [3]. Treatment with prednisolone (15 mg/day) was initiated. Raynaud's phenomenon, swelling of the hands, and slight myalgia in the upper extremities that had been present at the time of diagnosis improved within 3 months, but without any amelioration in the sensation in her face. In addition, not only immunosuppressive therapy but also both carbamazepine and phenobarbital which we tried, had no effect on the trigeminal neuropathy.

\section{Discussion}

In the present case, an isolated trigeminal sensory neuropathy with an intact corneal reflex and sparing of motor function occurred almost simultaneously with the onset of MCTD. The patient also manifested various clinical features that are diagnostic of MCTD: synovial thickening in the left shoulder joint, diffuse swelling of the hands, slight proximal myopathy with an increased CPK value, and a high titer of anti-RNP antibody.

Neurological abnormalities, of which trigeminal neuropathy has been the most frequently reported, make up approximately $10 \%$ of the pathological morbidity characteristic of MCTD [9] as shown in Table 1. Trigeminal neuropathy has also been reported together with symptoms of pain and numbness, and neurovascular headache of mild to moderate severity appears to be relatively common [10]; trigeminal neuropathy was thus the most common presentation of MCTD [11,12]. Several connective tissue diseases, including SLE, SSc, $\mathrm{PM} / \mathrm{DM}$, Sjögren's syndrome, and rheumatoid arthritis, have been

Table 1. Neurological abnormalities in MCTD.

Trigeminal neuropathy

Aseptic meningitis

Psychogenic disorder

Seizure

Headache

Peripheral neuropathy

Cerebellar ataxia

Modified consciousness

Transverse myelitis

Cubital and carpal tunnel syndrome associated with isolated trigeminal nerve neuropathy. This neuropathy seems to be sensory predominant, unilateral or bilateral, prodromal or late, occurring with or without pain, and having a variable prognosis. In addition, many patients with these other conditions appeared to have overlapping features of SLE, SSc, and PM/DM [13].

Other than trigeminal neuropathy, the neurological complications, that is, meningitis, cerebral ataxia, seizures, neuropsychosis, transverse myelitis, and peripheral neuropathy rarely occur in MCTD. Involvement of the sensory portion of the trigeminal nerve occurs in various disorders. Primary idiopathic sensory involvement is not associated with other neurological and systemic disorders, whereas secondary involvement is seen with tumors, connective tissue diseases, demyelinating disorders, trauma, and conditions secondary to toxic substances [14]. Peripheral neuropathy may accompany these disorders in several ways: symmetrical polyneuropathy, pure sensory peripheral neuropathy, acute neuropathy of the Guillain-Barré type, mononeuritis or mononeuritis multiplex, and cranial neuropathy. The motor part of the trigeminal nerve innervates the masseter and pterygoid masticatory muscles. Trigeminal neuralgia, a disorder of the sensory nucleus of the trigeminal nerve, causes severe shooting pain, which lasts a few seconds to several minutes, followed by a period during which no pain is left in areas affected by any of the branch territories. The pain results from stimulation of trigger zones, with the perioral and intraoral zones being the most common. The onset is usually sudden, and bouts tend to last for weeks or months before they remit spontaneously. Remissions may last a long time, but in most patients the disorder ultimately recurs.

The neuropathy in our patient was sensory, presumably because of a vascular lesion in or around the trigeminal ganglion. The question as to why the trigeminal sensory fibers are selectively involved in MCTD remains unanswered. Experimental evidence exists that blood vessels of the trigeminal ganglion are more permeable to proteins than is the blood-brain barrier. In the rat, horseradish peroxidase passes from venous blood into the extracellular space of the trigeminal ganglion within 2 minutes and penetrates the endoneurial space within 5 minutes. Selective trigeminal lesions may result from the entry into the ganglion of some neurotoxic serum factor [15]. The development of trigeminal neuropathy should prompt a careful search for underlying diseases. Among connective tissue diseases, MCTD should be considered a prime suspect. Although the overall prognosis of MCTD is favorable, patients require long-term follow-up before the prognosis of the associated trigeminal neuropathy can be determined. Clinical features of trigeminal sensory neuropathy occurring in association with MCTD are indistinguishable from those of this neuropathy occurring in isolation.

Formation of immune complex in the trigeminal nerve itself or in the sensory root may lead to isolated sensory dysfunction. Nerve biopsy from a patient with MCTD indicated vasculitis, fibrosis of nerve connective tissue, nerve infarcts, and axon and myelin damage $[16,17]$. Autopsy studies of patients with MCTD and trigeminal nerve dysfunction have not been reported. The existence of an isolated trigeminal sensory neuropathy in a symptomatic patient should thus be investigated carefully. Trigeminal sensory neuropathy may indeed be the harbinger of MCTD. Because trigeminal neuropathy precedes other features of MCTD, testing patients with atypical trigeminal neuropathy for the presence of anti-RNP antibodies to identify a subset of patients with both systemic disease and a potential to respond to immunosuppressive therapy may be useful. Elucidation of the mechanisms of development of MCTD will require additional pathological studies, and rheumatologists should be aware that 
trigeminal neuropathy may rarely be the presenting manifestation of MCTD.

Trigeminal sensory neuropathic complaints in patients with connective tissue disease usually begin with sudden onset of unilateral or bilateral facial numbness like the present case, with or without pain or paresthesia. In MCTD, this symptom would antedate the first indication of several connective tissue diseases, but generally follows the later by a matter of months. Trigeminal neuropathy may have to receive more attention than all the other neurologic sequelae of MCTD. The present case suggests that MCTD should be considered from a huge cases of isolated trigeminal neuropathy in cases where the diagnosis remains unclear.

\section{Acknowledgment}

The authors would like to thank Dr. Keigo Doufuku, Takachiho Municipal Hospital, Takachiho, Miyazaki, Japan, for his invaluable contribution and participation in the present case. The authors also appreciate Shinji Sato, MD, PhD, Department of Internal Medicine, Division of Rheumatology, Tokai University School of Medicine, Isehara, Kanagawa, Japan, for his helpful cooperation in analyzing anti-140-kDa polypeptide (CADM-140) antibody.

\section{References}

1. Tani C, Carli L, Vagnani S, Talarico R, Baldini C, et al. (2014) The diagnosis and classification of mixed connective tissue disease. J Autoimmun 48-49: 46-9. [Crossref]

2. Gryga K, Milewski M, Zolcinski M, Dyczek A, Musial J (2009) Anti-Ku autoantibodies: series of 5 cases. Pol Arch Med Wewn 119: 95-97. [Crossref]

3. Alarcon-Segovia D, Villareal M (1987) Classification and diagnostic criteria for mixed connective tissue disease. In: Kasukawa R, Sharp GC (Eds.), Mixed Connective Tissue Disease and Antinuclear Antibodies. Amsterdam: Elsevier; 33-40.

4. Castelino FV, Varga J (2010) Interstitial lung disease in connective tissue diseases: evolving concepts of pathogenesis and management. Arthritis Res Ther 12: 213. [Crossref]
5. Gutsche M, Rosen GD, Swigris JJ (2012) Connective Tissue Disease-associated Interstitial Lung Disease: A review. Curr Respir Care Rep 1: 224-232. [Crossref]

6. Papadimitraki ED1, Kyrmizakis DE, Kritikos I, Boumpas DT (2004) Ear-nose-throat manifestations of autoimmune rheumatic diseases. Clin Exp Rheumatol 22: 485-494. [Crossref]

7. Hojaili B, Barland P (2006) Trigeminal neuralgia as the first manifestation of mixed connective tissue disorder. J Clin Rheumatol 12: 145-147. [Crossref]

8. Cruccu G, Pennisi EM, Antonini G, Biassiotta A, di Stefano G, et al. (2014) Trigeminal isolated sensory neuropathy (TISN) and Fosmn syndrome: despite a dissimilar disease course do they share common pathophysiological mechanisms? BMC Neurol 14: 248 57. [Crossref]

9. Bennett RM, Bong DM, Spargo BH (1978) Neuropsychiatric problems in mixed connective tissue disease. Am J Med 65: 955-962. [Crossref]

10. Klasser GD, Balasubramaniam R, Epstein J (2007) Topical review-connective tissue diseases: orofacial manifestations including pain. JOrofac Pain 21: 171-184. [Crossref]

11. Searles RP, Mladinich EK, Messner RP (1978) Isolated trigeminal sensory neuropathy: early manifestation of mixed connective tissue disease. Neurology 28: 1286-1289. [Crossref]

12. Varga E, Field EA, Tyldesley WR (1990) Orofacial manifestations of mixed connective tissue disease. Br Dent $J$ 168: 330-331. [Crossref]

13. Vincent FM, Van Houzen RN (1980) Trigeminal sensory neuropathy and bilateral carpal tunnel syndrome: the initial manifestation of mixed connective tissue disease. $J$ Neurol Neurosurg Psychiatry 43: 458-460. [Crossref]

14. Hamoir B, Giroux M, Outteryck O, Launay D, Vermersch P (2014) Mixed connective tissue disease presenting as trigeminal neuropathy. Acta Neurol Belg 114: 245-246. [Crossref]

15. Lecky BR, Hughes RA, Murray NM (1987) Trigeminal sensory neuropathy. A study of 22 cases. Brain 110: 1463-1485. [Crossref]

16. Currie DM, Bradshaw DC (1979) Polyneuropathy in mixed connective tissue disease presenting as progressive systemic sclerosis (scleroderma): case report and literature review (abstract). Arch Phys Med Rehabil 60: 594.

17. Nascimento IS, Bonfá E, de Carvalho JF, Saad CG, Vendramini MB, et al. (2010) Clues for previously undiagnosed connective tissue disease in patients with trigeminal neuralgia. J Clin Rheumatol 16: 205-208. [Crossref]

Copyright: (C2015 Nakamura T. This is an open-access article distributed under the terms of the Creative Commons Attribution License, which permits unrestricted use, distribution, and reproduction in any medium, provided the original author and source are credited. 